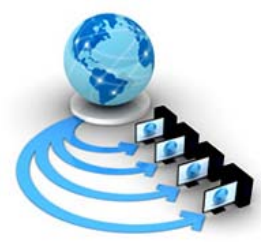

Volume 12, No. 1, January-February 2021

REVIEW ARTICLE

\title{
REQUIREMENT ANALYSIS OF NODE DEPLOYMENT IN MOBILE BASED SENSOR NETWORK
}

\author{
Putri Kevin \\ Department of Computer Science, \\ Brahijaya University,Indonesia
}

\author{
Dian viely \\ Department of Computer Science, \\ Brahijaya University,Indonesia
}

\begin{abstract}
The movement assisted sensor deployment is the most common design issue in mobile design. Various models, assumptions, goals, and shortcomings are identified, and formulas are mentioned. A taxonomy of motion-assisted sensor deployment algorithms that captures fundamental variations between current solutions is introduced. Six approaches are identified; they use a particular theory to shift the node from the original position to the target position. The comparison of the self-deployment algorithm and classes is discussed in detail in this paper.
\end{abstract}

Keywords: mobile sensor network, Deployment, Dense, anchor, WSN

\section{INTRODUCTION}

The wireless sensor network (WSN) consists of sensor nodes (or sensors), which communicate over through wireless channels to detect the region of interest (ROI). Coverage is a troubling issue in ROI. To avoid convergence hole, sensors may not be placed too far from each other. The number of sensors in ROI determines network topology, which would impact connectivity, cost, and lifetime [1-4]. Deterministic deployment is not feasible where human intervention is not possible. Random sensor deployment is a considerable solution that results in an uncontrolled converge degree of ROI. Coverage may be increased either by deploying sensors with more comprehensive sensing ranges or deploying a vast number of sensors in a specific area to ensure maximum coverage. With the ability to on ones on WSN are capable of self-deploy and self-repair.

Sensor relocation and movement assistant sensor deployment are two different issues. In sensor relocation, dynamic repositioning of the nodes while the network is in operation is required to enhance its efficiency. For example, the failure of many sensors in the vicinity of the sink due to their batteries' exhaustion can break down communication paths in the network [5-6]. In the worst case, the network will be partitioned into several sub-networks and become dysfunctional. In this situation, specific redundant sensors from other ROI areas may be detected and transferred to replace dead sensors to increase the network's existence. Mobile sensors can be self-configured after early implementation in motion-assisted sensor deployments to accomplish improved configuration and expand the network's efficiency. Ghosh and Das [1] address and compare multiple node implementation algorithms for both static and mobile as well as hybrid sensor networks.

\section{TAXONOMY}

We classify the identified strategies into six classes, which are: 1) Virtual Forces-Based Method, 2) Pattern-Based Method, 3) Grid-Quorum Method, 4) Computational Geometry Method 5) Fuzzy Logic Method, and 6) Metaheuristic-Based Method. We compute matrices for different deployment algorithm. CM: Coverage Model may be either binary (b) or Probabilistic (P).
Degree of coverage-CD: Reflects the degree of coverage that the algorithm may ensure; it may be singular (S) or controlled (T) [7].

Distributed vs Centralized (D/C): Determines whether an algorithm is performed by each node in the network or only by a single node [8].

$\boldsymbol{R} \boldsymbol{c}$ vs Rs: Defines the relationship between the communication range and sensing Range, NDR:

No Direct Relation.

Primary Objective. Defines the key goal that the algorithm is attempting to accomplish.

Termination condition: It states the convergence of the algorithm.At each iteration, The algorithm contrasts the real degree of coverage with that of the prefixed one. Typically this condition is used in Centralized algorithms [9-11].

A number of iterations: The algorithm moves for a number of iterations.

Stability: Node is stable and must stop moving if sensor distance is smaller than the threshold for known iterations in a distributed network.

Oscillation: Algorithm terminated if nodes have oscillation at the same location.

Balanced load: The algorithm stops when the number of nodes is on each cluster in the grid is balanced.

Local hole coverage: Each node determines its coverage hole size and attempts to repair it [12].

Efficiency and effectiveness metrics: Complexity, overhead coordination, speed of convergence, and the number of moves of all mobile sensors

\section{STATIC METHODS}

In the flat method, all sensor nodes are the same. The flat method is further divided into sub approaches.

\section{A. Virtual forces}

In a virtual force-based method, the sensor behaves as an electromagnetic particle. This concept is used to relocate the sensor. Inter sensor force may be attractive and repulsive. Each sensor is assumed to know its position and all of its neighbors. If sensors are close to each other, they exert the repulsive force computed by a predetermined threshold. This keeps the overlapping coverage area minimum if sensors are too far from each other; they exert an attractive force on each 
other; this maintains a global uniform sensor placement. The cumulative force determines the sensor's final movement that the sensor is due to the sensors in its neighborhood [13-15].

\section{B. Virtual forces algorithm (VFA)}

VFA algorithm is motivated by disk packing theory and VFF from robotics. It strives to maximize the sensor coverage by a combination of attractive and repulsive forces. During the VFA algorithm execution, the sensors do not physically move, but virtual motion paths for randomly located sensors are calculated. When the sensor positions are marked, a one-time movement is carried out to redeploy the sensors at these positions. VFA is a cluster-based network sensor architecture where the cluster head is responsible for running the VFA algorithm and controlling sensors' one-time movement to the target location.

Assumption: ROI is the 2D grid. All sensor communicates with the cluster head, after arbitrary deployment. A binary and Probabilistic coverage model is used.

Principle: The operating principle is the VF method.

Advantages: VFA has simple communication, fast \& Improve coverage, and control coverage degree by a threshold. A one-time movement to a final point.

Disadvantages: In VFA, simultaneous movement of nodes may have created collision. Wa and Wr are the parameters that affect the efficiency of the algorithm. Cluster-based network architecture is not always feasible. Computation of the virtual paths results in high energy consumption. When a movement decision is made, no energy level is assumable.

Variants and ameliorations: IVFA (Improved Virtual Force Algorithm) explains the boundary effect, effective VFA distance, unnecessary movements, and convergence issue of VFA in a given RO. EVFA (Exponential Virtual Force Algorithm) improves the convergence speed of VFA. EVFA (Energy-considered Virtual Force Algorithm) elongating the lifespan of the WSN. CPVF (Connectivity Preserving Virtual Force) ensures network connectivity. TIVFA (Target Involved Virtual Force Algorithm) improves coverage and multi-target tracking [16-17].

\section{Enhanced self-deployed methods (ESD)}

ESD is a distributed algorithm that aims to overcome the coverage hole issue and network division.

Assumptions: The binary sensor coverage model is assumed.

Principle: DC is a virtual point that exerts a force on node and Dc point specified in ESD. Around the same time, repulsive force occurs between nodes to ensure sufficient space between nodes to prevent overlap coverage [18].

Advantages: Improve coverage network recovery. It allows a fast recovery of the network when nodes across the base station crash.

Disadvantage: Network deployment should be thick to guarantee network coverage boundaries because DC attracts nodes towards itself. The efficiency depends on the parameters $l r$ and $l a$. Energy level not considered.

\section{BODVFA}

A randomized back-off delay period is added to address the simultaneous movement of sensors in neighbours, leading to excessive undue movement and waste more sensor resources. The sensors are randomly scattered in the RoI, have the same detection communication range and initial energy. Sensor Equip with GPS to locate itself and terrains. principle: BIDVFA explains that sensor movement consumes more energy; the movement needs to be minimized. The randomized delay time is calculated when all sensor innetwork at iteration, and this delay time help resolve the sensor's simultaneous movement.

Advantages: It improves coverage, controls the coverage degree \& eliminates the coverage hols, and limits nodes' movement to preserve energy [19].

Disadvantage: The flow of information between nodes before the movement consumes a considerable amount of energy. The back delay off in iteration requires high computations.

\section{E. Computational geometry-based methods}

Geometrical computation is used to discover the coverage holes in ROI. Voronoi diagram and Delaunay triangulation are two geometry-based approaches. The Voronoi diagram represents the proximity information about geometric nodes. The Voronoi diagram of a set of nodes splits space into a polygon. Based on the sensors' location, the Voronoi network diagram is built, and the decision whether the sensors need to be repositioned or Stay would be made based on the diagram.

VEC, VOR, and MiniMax protocols based on Voronoi aim to obtain a uniform distribution of the RoI sensors by moving the sensors from dense regions to scattered regions on the Voronoi diagram. In [8] Biding protocol is proposed, which uses a static sensor to identify coverage holes by Voronoi means. Then the mobile sensors will be relocated to fill the holes found by the static ones. Three algorithms are developed on a multiplicatively weighted Voronoi diagram: MWV, MWP, and MDW. When all sensors are homogenous, every point in ROI has the same MDV algorithm as a VOR strategy. $\mathrm{MW}$-Voronoi is a case of non-uniform coverage with nonidentical sensors.

VOR: Voronoi based algorithm: VOR pulls the sensor to its maximum coverage hole. If a sensor senses the presence of coverage holes in VOR, it pushes towards its farthest vertex of Voronoi. The moving distance is restricted to half of the communication range to avoid communication limitations.

Advantage: Initial coverage improves.

Disadvantage: useless in dealing with large coverage holes and oscillation moves on.

\section{F. Fuzzy optimization}

A FOA uses the fuzzy logic to control the node movement based on a distributed algorithm. By applying this fuzzy optimization process to each mobile sensor, oscillation and unwanted motions are effectively prevented, and rapid distribution is accomplished.

Advantages: Node Collision is avoided due to Coherence time. Coverage improves by FOA [20].

Principle: A given amount o sensor deployed in sequre region, two terms are considered, 1) determine next movement 2) no of sensors in neighbour and average Ecuadorian distance between the sensor and its adjacent nodes

Disadvantages: May not take into account the existence of an obstacle.

\section{G. Fuzzy based approach}

Fuzzy logic is used to compute the movement of the mobile sensor in a distributed network

Assumption: Each node recognizes its position and detects the obstacle place in its path. The communication range is higher than the coverage [21]. The binary model is used. 
Principle: Collecting and computing neighbor information. Computing vector movement. Nodes find out the neighboring node in its coverage range and detect obstacles. There is two input, one is no of mobile nodes, and second is sensing shadow length on an obstacle. Each node move before the adjacent node, then the sensor finds out the vector movement with respect to neighbour. After that, by specifying the destination, mobile nodes' lowest priority to move to a new destination [22].

Advantages: Better coverage, obstacle, and boundaries should be taken into account. Collision is avoided due to movement priority.

Disadvantages: Performance affected by new topologies.

\section{H. Metaheuristic based approach}

To set the location, orientation, and speed of a mobile sensor movement, algorithms leverage the strength of metaheuristics.GA, PSO AC, and SA are classes of this algorithm. First, a population of candidate solutions for the problem is generated. Each soul is identified by the genome, consisting of one or more chromosomes, represented by the speed, orientation, and target of the sensor's movement. This genome is used in the fitness function [23]. A distributed algorithm is used to show up the sensor in the fitness function, which uses the node and its adjacent orientation. The algorithm assumes a dense initial deployment to ensure coverage of the entire deployment area.ABC(Artificial bee colony ) based on honey bee etiquette.

\section{LODICO}

LODICO is a distributed algorithm.

Assumption: Each node identify its orientation \& position. Thick Initial deployment. Rc>3Rs.

Principle: LODICO runs during numbers of iteration on each node. The node goes through three stages. a) Planning, b) Determining, and c) Movement. In the first stage, Node exchange data with others node within its coverage region. In computation, the target position is identified, the node relocates, and the node [24].

broadcasts its new position and prepares for the next iteration.

Advantages: Improve coverage. Reduction of pass over distance by the restriction of the research space for each node. Nodes movement is based on updated neighborhood proximity information.

Disadvantages: High complexity. The quality of the obtained solutions depends on many parameters (e.g., number of iterations and GA related parameters). Nodes' energy is not taken into account.

\section{J. Force based genetic algorithm FGA}

FGA is a distributed algorithm principled on intermolecular forces in Physics. Each node shares genetic (Speed and Direction)information with the neighbour within its communication range [25].

Assumptions: Each node locates in 6 directions and knows its location.ROI divided into a virtual mesh. Every node collects genetic information about its neighbours and uses FGA to compute its next speed and movement. To determine the optimal solution for the next movement stage, a fitness function is used.

Advantages: Uniform Distribution of nodes and node failure and Obstacle In ROI taken into consideration.
Disadvantages: Node energy is not taken into account, and FGA is complicated.

\section{HIERARCHICAL METHODS}

This class includes coverage pattern based and grid quorum based approaches.

\section{A. Coverage based approach}

Depending on the coverage pattern, the final locations where the mobile sensors will be relocated are pre-calculated based on a pre-selected coverage pattern that will guarantee the whole network's optimal coverage and connectivity. The ROI is divided into grids in coverage form. Triangular lattice, square grid, and hexagonal grid are commonly used. Triangular lattice is best because of having less overlapping regions. Hexagonal is worst because it has the largest overlapping area. The square grid is good in terms of performance. For a highly dense network, a small size grid helps in reducing coverage holes. Lam and lim proposed the distributed algorithm know as isogrid, for uniform coverage of ROI uses a triangular pattern. Snap and spread use a hexagonal pattern to construct uniform mesh. Push and pull is an improved version of S\&S. They are translating the problem of node relocation into a matching problem based on a regular pattern that can guarantee network connectivity.

\section{B. Crystal Lattice permutation CLP}

CLP is a distributed algorithm driven by two factors: Finding that most VFA-controlled node traces are curves. The second motivation is that node's best locations will be the vertexes of the hexagonal topology.

Assumptions: Sensors are uniformly deployed in ROI and have the same locomotive capabilities. Each node has a GPS to know its location. A unique node is assigned as an initial seed. The sensor coverage model is binary [26-27].

Operating Principle: All the nodes begin in the neighbouring state. One seed automatically reaches the seed state, considering it to be the initial node. E.g., a node with an ID zero can be assigned as the initial node. The initial seed selects at most six adjacent nodes to shift to the desired hexagon location. When the node becomes the initial seed, it starts to collect its neighbouring information. For each hex location, the shortest distance node is greedily selected from the array. The seed sends its destination to the chosen node. If the node has been picked simultaneously as other seeds, it responds with a "Reject Message."

Advantages: uniform ROI coverage and seed failure were taken into account.No of a move are limited compared to other move-in VFA.

Weaknesses: Initial deployment is dense to follow up on hexagonal construction. It is an omission to obstacles. At the initial movement, energy levels are not considered.

\section{Push and pull}

$\mathrm{P} \& \mathrm{P}$ is a distributed algorithm based on an automatic computing model. P\&P aims to allow the sensors to form a hexagonal tiling, full RoI coverage, and the connected network deployment.

Assumptions: In P\&P, some sensors adhere to the hexagonal grid points and let others uniformly distributed over the whole RoI. According to the P\&P algorithm, the sensors are engaged in four simple activities mention below. 
Snaped: In the beginning, the sensor may give a tile portion creation by snaping itself at the current position at the randomly selected time. It chooses the center of the position of the hexagon. All sensors in its neighbour reply with a message of a role as slave or snaped. The snaped sensor conducts the discovery of neighbor, enabling the snapped sensor to be aware of empty spaces location in its neighbourhood. Then the snapped sensor picks six of the nearest slave sensors to relocate to exposed locations. After slave sensors have reached their places, and launch their Snap operation [28].

Push: After the Snap activity termination, the snapped sensor encircled by slave sensors located inside its tile portion. According to a Push activity, the snapped sensor appraises the chance to move some of its slaves to neighbour hexagons to a uniform distribution of unnecessary sensors.

Pull: If no sensor can be obtained from its snapped neighbours, the Pull operation will begin. When a sensor receives a Hole info message and does not have a slave to drive into the coverage hole, it participates in the Pull operation by transmitting it when necessary [29-33].

Merger: Sensors act as a starter and generates several portions of tiles with different orientations. Merge operation consists of a fusion of two or more adjacent parts of the tiles [34] in such a way as a section with the oldest start time will be adopted other parts of it.

Advantages: Uniform coverage without initial topology and ROI coverage with less no of nodes. One-time movement obstructs oscillation \& consumes less energy.

Disadvantages: Omission to obstacle and hexagonal grid have large overlapping regions.

\section{GRID quorum based approach}

Deploying the sensor leads to a load balancing problem as in conventional parallel processing. Each area represents a processor, and no of the sensor represents a load. ROI is divided into grid cells through the cluster. Each cluster represents a small area and cluster heads that share information with the adjacent cluster [35-36]. The grid quorum base method does not identify the target location. The scan operation is used to compute the average load and enumerate the underload and overload cluster. The load is moving from overload to underload under the limitation of reducing transvered distance.

SMART: Scan-based Movement-Assisted sensor deployment: SMART trade-in shifting of sensors from unbalanced to a balanced state.

Assumption: Dense deployment and each node find its position and node have two communication ranges: intracluster and intracluster [37].

Principle: Sensor network divided into an nxn 2D mesh cluster. Each cluster has its head that covers a small region. Each cluster head is in charge of collecting information from adjacent nodes. $1^{\text {st }}$ cluster position and $2^{\text {nd }}$ no of sensors.

Advantages: Swift convergence and uniform distribution of nodes.

Disadvantages: To guarantee the presence of nodes, the first deployment must be populated. Cluster Head failure doest not consider

\section{E. CORONA radious scanning based approach}

Corona radius is a distributed algorithm that assumes the unequal distribution of energy consumption in the network. Nodes near the sink use their energy more swiftly. These nodes are requested by the other nodes of the network to onwards the collected information to the sink, which will speed up their energy consumption and increase threats to the nodes' failure [38]. Corona Radius seeks to find a non-uniform deployment strategy that would extend network life, thus minimizing sensor movement.

Assumption: Network is divided into sections and corona virtually. Sensors deployed massively; node density depends upon corona. The communication range is equal to corona wilderness.

Principle: Number of sensors per corona balances by corona scan. In radius-scan, sensors are reallocated to sections following the desire density of sensors.

Advantages: The non-uniform distribution ensures fault distribution and extends the network lifespan.

Disadvantages: Initial deployment must be populated. It does not consider the communication hole and failure of the head of each sector.

\section{MISCELLANEOUS METHODS}

We find a few literature methods that do not share their principle theory with the six classes discussed earlier. In this segment, we will cover these methods.

\section{A. Event-driven deployment by self-organized map}

The objective is to disperse the sensor so that the distributed sensor's density corresponds to the probability density of the event to be detected. To address the coverage problem, the concept of a Self-organizing Map is explored. SOM is an approach based on an artificial neural network in unsupervised learning.

Assumptions: All sensors are similar. The expected event distribution is considered to be stationary. The binary disk model is viewed as a sensor coverage model [39].

principle: Sample points is bringing about to reflect events' distribution. The algorithm begins with a random initial orientation of the sensors, accompanied by an iterative adaptive rearrangement method by a small shift of the sensors. Each iteration attention on a single sample and the sensor nearest to that sample is identified as "winner." The winner will be able to step closer to the sample. This process is repeated until no further meaningful movement is attainable.

Advantages: Effective in uniform distribution of events. The probability density for events to be sensed is considered. Initial coverage improving [40-41].

Disadvantages: Highly complicated. Node energy is not taken into account.

\section{B. ZONE based relocation}

ZONE is a protocol for the relocation of sensors based on limited flooding techniques. ZONER can accurately detect deployed extra sensors and move them to replace failed sensors without altering the network's topology.

Assumptions: Each node knows its location by a global coordinate system. Linked network to redundant nodes. Omnidirectional antenna. $R c \geq R s$. The network remains connected if any node can malfunction

principle: ZONER has two zones, 1) Register zone. Each redundant node broadcasts its presence by using the limited flooding methods across the whole network. All nodes in the flood region will be informed. (2) A horizontal zone or a request zone where a research process is used to replace a failed node in another region of the network, the research algorithm is stopped until a redundant node is in a vertical 
zone, so it is transferred to the correct location a moving direction.

Advantages: Contend to maintain the topology of the network and coverage improvement.

Weaknesses: When choosing a node, node energy is not considered. The issue of void areas continues. The flooding method is expensive because of messages exchanged.

\section{Market competitionbased approach}

A distributed network controls the connectivity \& coverage issue.

Assumptions: The target area is a rectangular region with unneeded nodes. All nodes embedded with GPS. To minimize the deployment cost, node deployment is an alloy of a static and mobile node, power of static nodes higher than the mobile nodes. The whole network is divided into a subnetwork, which they can't communicate with each other [42].

Principle: The network includes both static \& mobile nodes. Static nodes act as a wide enterprise and benefit of occupying resources. Mobile nodes act as small companies where the unallocated services will be shared based on the competitive mechanism between them to create a "quasi-static coverage of sensors," the new dynamic sensors will engage in the expansion process.

Advantages: Reduce the complexity of exchange messages. Reduce distance travel by sensors.

Disadvantage: Non-complex deployment.

\section{OPEN RESEARCH STATEMENT}

We highlight open research topics in this section and describe the challenges involved and update current work and preliminary findings [43].

\section{A. Sensor network coverage}

Binary and probabilistic models are mostly used in the coverage model. Mostly self-deployment sensor used is a binary model. While the binary model streamlines the analysis, in many cases it might not be realistic since it fails to apprehend the sensor's casual nature. The binary model, uncertainty, and imprecision correlated with the sensor reading. The Probalistic model more realistically captures the sensor's actions; the cooperation between sensors is not abused. Moreover, it remains limited. Sensor reliability should be taken into account in this model [44]. The transferable Confidence Model (TBM) was established in a recent study. This coverage model not only considers the fault related to sensor readings, but it can also be conveniently expanded to cover problems related to deployment, just as sensor reliability. The above literature suggested that the binary coverage model is strictly associated with the sensor selfdeployment approach. Realistic coverage models should be thinking about in future research in this field.

\section{B. Sensor Communication}

The binary disk model, also known as UGD (unit disk graph), is generally used in the study of connectivity of WSN. This model explicitly approaches the problem of network connectivity from a geometric viewpoint, which simplifies the analysis. If all nodes are homogenous, this model is not interpreted for obstacles, and weather conditions may block the signal propagation. If a convex ROI is covered with multiple sensors, these sensors' communication graph is connected when $R c \geq 2 R s$ [45]. Under this condition, WSN must only be designed to ensure coverage to accommodate both coverage and connectivity. Connectivity is not an issue if the Rc transmission range is much longer than Rs. This result is generalized in the case of $\mathrm{k}$-coverage. When $R c \geq 2 R s$, $\mathrm{k}$ coverage means k connectivity. The coverage and connectivity are still unresolved in the probabilistic model.

\section{WSN self deployment}

Most research papers focused on 2D ROI. With the disclosure of the 3D ROI application scheme like underwater surveillance, addressing self-deployment problems in 3D has been a must. The 3D volume (e.g., underwater sensor deployment) and 3D surface (e.g., mountain surface deployment) are reviewed. The $3 \mathrm{D}$ coverage issue is minimizing the geometric problem from 3D to 2D space. Deployment of 3D-dimensional mobile sensor networks focused on virtual force algorithm. In, authors adapt the traditional VFA to 3D space, whereas utilize fluid dynamics, Where WSN is fluid, and the node is considered as a charged particle. Connectivity is the main issue in the $3 \mathrm{D}$ deployment zone.

\section{Pathplanning}

In WSN, path planning was scrutinized to plan the movement of data mules that collect data from a stationary node. The constructive criticism on the self-deployment algorithm is that the supposition statement makes on sensor nodes' ability, specifically the sensor nodes' ability to move to every position required to move. When obstacles need to be an omission, a significant adverse effect on energy usage can be expected in all self-deployment protocols. Path planning should be considered in designing the self-deployment algorithm in the future [46].

\section{CONCLUSION AND FUTURE WORK}

In MWSN, where all nodes switch around and change their location after deployment to revamp network performance. This research brings in a movement assisted sensor deployment algorithm taxonomy that apprehends the fundamental discrepancy among existing algorithms. We also divided the current self-deployment systems into six groups. We focus on a number of motion-assisted sensor implementation algorithms proposed and researched by researchers and highlight their strengths and shortcomings. In open research, we consider the two main module issues: communication and sensor coverage model. To solve the realistic model, we visualize the $3 d$ Self-deployment, which requires intensifying attention from research. Future work on the phase of self-deployment, path planning is to be considered at the design phase.

\section{REFERENCES}

[1] S. S. Kulkarni, "TDMA Services for Sensor Networks,"Proc. 24th Int'l. Conf. Distrib. Comp. Sys. Wksps., Mar.2004, pp. 604-09.

[2] W. Ye, J. Heidemann, and D. Estrin, "Medium Access Control with Coordinated Adaptive Sleeping for Wireless Sensor Networks,” IEEE/ACM Trans. Net., vol. 12, no. 3, June 2004, pp. 493-506.

[3] A. El-Hoiydi, "Spatial TDMA and CSMA with Preamble Sampling for Low Power Ad Hoc Wireless Sensor Networks,” Proc. ISCC 2002, July 2002, pp. 685-92. 
[4] Ahmad, T., Li, X. J., \& Seet, B. C. (2017). Parametric loop division for 3D localization in wireless sensor networks. Sensors, 17(7), 1697.

[5] V. Rajendran, K. Obraczka, and J. J. Garcia-Luna-Aceves, "Energy-Efficient, Collision-Free Medium Access Control for Wireless Sensor Networks,” Proc. ACM SenSys '03, Los Angeles, CA, Nov. 2003, pp. 181-92.

[6] L. Bao and J. J. Garcia-Luna-Aceves, "A New Approach to Channel Access Scheduling for Ad Hoc Networks," 7th Ann. Int'l. Conf. Mobile Comp. and Net., 2001, pp. 21021.

[7] Ahmad, Tanveer, Xue Jun Li, and Boon-Chong Seet. "A self-calibrated centroid localization algorithm for indoor ZigBee WSNs." In 2016 8th IEEE International Conference on Communication Software and Networks (ICCSN), pp. 455-461. IEEE, 2016.

[8] Dawadi, B. R., Rawat, D. B., Joshi, S. R., \& Keitsch, M. M. (2020). Towards energy efficiency and green network infrastructure deployment in Nepal using software defined IPv6 network paradigm. The Electronic Journal of Information Systems in Developing Countries, 86(1), e12114.

[9] Ahmad, Tanveer, Xue Jun Li, and Boon-Chong Seet. "3D localization based on parametric loop division and subdivision surfaces for wireless sensor networks." In 2016 25th Wireless and Optical Communication Conference (WOCC), pp. 1-6. IEEE, 2016.

[10] K. Jamieson, H. Balakrishnan, and Y. C. Tay, "Sift: A MAC Protocol for Event-Driven Wireless Sensor Networks,” MIT Lab. Comp. Sci., Tech. rep. 894, May 2003, available

http://www.lcs.mit.edu/publications/pubs/pdf/MITLCSTR-894.pdf

[11] Y. C. Tay, K. Jamieson, and H. Balakrishnan, "CollisionMinimizing CSMA and Its Applications to Wireless Sensor Networks,” IEEE JSAC, vol. 22, no. 6, Aug. 2004, pp. 1048-57.

[12] Ahmad, Tanveer, Xue Jun Li, and Boon-Chong Seet. "3D localization using social network analysis for wireless sensor networks." In 2018 IEEE 3rd international conference on communication and information systems (ICCIS), pp. 88-92. IEEE, 2018.

[13] Chen, Hongyang, Pei Huang, Marcelo Martins, Hing Cheung So, and Kaoru Sezaki. "Novel centroid localization algorithm for three-dimensional wireless sensor networks." In 2008 4th International Conference on Wireless Communications, Networking and Mobile Computing, pp. 1-4. IEEE, 2008.

[14] Chen, Kai, Zhong-hua Wang, Mei Lin, and Min Yu. "An improved DV-Hop localization algorithm for wireless sensor networks." (2010): 255-259.

[15] zeng Wang, Ji, and Hongxu Jin. "Improvement on APIT localization algorithms for wireless sensor networks." In 2009 International Conference on Networks Security, Wireless Communications and Trusted Computing, vol. 1, pp. 719-723. IEEE, 2009.

[16] Ahmad, Tanveer, Xue Jun Li, and Boon-Chong Seet. "Noise Reduction Scheme for Parametric Loop Division 3D Wireless Localization Algorithm Based on Extended Kalman Filtering." Journal of Sensor and Actuator Networks 8, no. 2 (2019): 24.

[17] Ahmad, Tanveer, Xue Jun Li, and Boon-Chong Seet. "Fuzzy-Logic Based Localization for Mobile Sensor Networks." In 2019 2nd International Conference on Communication, Computing and Digital systems (CCODE), pp. 43-47. IEEE, 2019.

[18] G. Lu, B. Krishnamachari, and C. S. Raghavendra, “An Adaptive Energy-Efficient and Low-Latency MAC for
Data Gathering in Wireless Sensor Networks,” Proc. 18th Int'l. Parallel and Distrib. Processing Symp., Apr. 2004, p. 224.

[19] Guerrero, Esteban, H. G. Xiong, Qiang Gao, Gabriel Cova, Ricardo Ricardo, and J. Estévez. "ADAL: A distributed range-free localization algorithm based on a mobile beacon for wireless sensor networks." In 2009 International Conference on Ultra Modern Telecommunications \& Workshops, pp. 1-7. IEEE, 2009.

[20] Ahmad, Tanveer, Xue Jun Li, Boon-Chong Seet, and Juan-Carlos Cano. "Social Network Analysis Based Localization Technique with Clustered Closeness Centrality for 3D Wireless Sensor Networks." Electronics 9, no. 5 (2020): 738.

[21] hakila, R., and B. Paramasivan. "An improved range based localization using Whale Optimization Algorithm in underwater wireless sensor network." Journal of Ambient Intelligence and Humanized Computing (2020): 1-11.

[22] Ahmad, T. (2019). 3D Localization Techniques for Wireless Sensor Networks (Doctoral dissertation, Auckland University of Technology).

[23] R. Niu and P. Varshney, "Target location estimation in wireless sensor networks using binary data," in Proceedings of the 38th International Conference on Information Sciences and Systems, pp. 17-19, Princeton, NJ, USA, March 2004.

[24] Ahmad, T. (2019). 3D Localization Techniques for Wireless Sensor Networks (Doctoral dissertation, Auckland University of Technology).

[25] M. P. Michaelides and C. G. Panayiotou, "SNAP: fault tolerant event location estimation in sensor networks using binary data,” IEEE Transactions on Computers, vol. 58, no. 9, pp. 1185- 1197, 2009.

[26] Ahmad, T., Li, X. J., Wenchao, J., \& Ghaffar, A. (2020, September). Frugal Sensing: A Novel approach of Mobile Sensor Network Localization based on Fuzzy-Logic. In Proceedings of the ACM MobiArch 2020 The 15th Workshop on Mobility in the Evolving Internet Architecture (pp. 8-15).

[27] K. Lu, X. Xiang, D. Zhang, R. Mao, and Y. Feng, "Localization algorithm based on maximum a posteriori in wireless sensor networks," International Journal of Distributed Sensor Networks, vol. 2012, Article ID 260302, 7 pages, 2012.

[28] L. Cheng, C. D. Wu, Y. Z. Zhang, and Y. Wang, "Indoor robot localization based on wireless sensor networks," IEEE Transactions on Consumer Electronics, vol. 57, no. 3, pp. 1099-1104, 2011.

[29] Y. Wang, X. Wang, D. Wang, and D. P. Agrawal, "Range-free localization using expected hop progress in wireless sensor networks,” IEEE Transactions on Parallel and Distributed Systems, vol. 20, no. 10, pp. 1540-1552, 2009.

[30] H. Xu, Y. Tu, W. Xiao, Y. Mao, and T. Shen, “An archimedes curve-based mobile anchor node localization algorithm in wireless sensor networks," in Proceedings of the 8th World Congress on Intelligent Control and Automation (WCICA '10), pp. 6993-6997, Jinan, China, July 2010.

[31] J. Lee, W. Chung, and E. Kim, "Robust DV-Hop algorithm for localization in wireless sensor network," in Proceedings of the International Conference on Control, Automation and Systems, pp. 2506-2509, Gyeonggi-do, South Korea, October 2010.

[32] Ware, R.; Lad, F. Approximating the Distribution for Sum of Product of Normal Variables; Research report; the Mathematics and Statistics department at Canterbury University: Christchurch, New Zealand, 2003. 
[33] Wen, C.-Y.; Hsiao, Y.-C. Decentralized anchor-free localization for wireless ad-hoc sensor networks. In Proceedings of IEEE International Conference on Systems, Man, and Cybernetics, Singapore, October 2008; pp. 2777-2785.

[34] Roberts, G.; Gelman, A.; Gilks, W. Weak Convergence and Optimal Scaling of Random Walk Metropolis Algorithms. Technical Report; University of Cambridge: Cambridge, UK, 1994.

[35] Chan, Fu and Chih-yu “Adaptive AoA/ToA localziation using fuzzy particle for mobile WSNs” in 2011 IEEE $73^{\text {rd }}$ VTC Spring 2011.

[36] Liu, Q.; Ihler, A. T.; Smyth, P. Particle filtered MCMCMLE with connections to contrastive divergence. In Proceedings of the 27 th International Conference on Machine Learning, Haifa, Israel, June 2010; pp. 1-8.

[37] Canovas, J. P.; LeBlanc, K.; Saffiotti, A. Robust multirobot object localisation using fuzzy logic. In Proceedings of 2004 Int. Robocup Symposium, Lisbon, Portugal, July 2004; pp. 247-261.

[38] Liu, C.; Wu, K.; He, T. Sensor localization with ring overlapping based on comparison of received signal strength indicator. In Proceedings of IEEE Mobile Ad-hoc and Sensor Systems (MASS'04), Fort Lauderdale, FL, USA, October 2004; pp. 516-518.

[39] Doucet, A.; de Freitas, N.; Gordon, N. Sequential Monte Carlo Methods in Practice; Springer-Verlag: New York, NY, USA, 2001.

[40] Chib, S.; Greenberg, E. Understanding the MetropolisHastings algorithm. Amer. Statist. 1995, 49, 327-335.
[41] Chintalapudi, K.K.; Dhariwal, A.; Govindan, R.; Sukhatme, G. Ad-hoc localization using ranging and sectoring. In Proceedings of INFOCOM, Hong Kong, China, 7-11 March 2004; pp. 2662-2672.

[42] Ihler, A.T.; Fisher, J.W.; Moses, R.L.; Willsky, A.S. Nonparametric belief propagation for self-localization of sensor networks. IEEE J. Sel. Areas Commun. 2005,

[43] Wu, Jiawei, Jinming Yu, Aijun Ou, Yiming Wu, and Wuiun Xu. "RCDV-Hop Localization Algorithm for WSN." In 2012 8th International Conference on Wireless Communications, Networking and Mobile Computing, pp. 1-4. IEEE, 2012.

[44] Chen, W.; Mei, T.; Meng, M. Q.; Liang, H.; Liu, Y.; Li, Y.; Li, S. Localization algorithm based on a spring model (LASM) for large scale wireless sensor networks. Sensors 2008, 3, 1797-1818.

[45] Savvides, A.; Park, H.; Srivastava, M. The bits and flops of the N-hop multilateration primitive for node localization problems. In Proceedings of the First ACM International Workshop on Sensor Networks and Applications, Atlanta, GA, USA, September 2002; pp. 112-121.

[46] Savvides, A.; Han, C.-C.; Srivastava, M. B. Dynamic fine-grained localization in ad-hoc networks of sensors. In Proceedings of the 7th annual international conference on Mobile computing and networking, Rome, Italy, 16-21 July 2001; pp. 166-179. 